\title{
AVALIAÇÃO DE CLONES DE BANANA CAVENDISH
}

\author{
Evaluation of cavendish banana clones
}

\author{
Sebastião de Oliveira e Silva ${ }^{1}$, Elias Teixeira Pires ${ }^{2}$, Rosa Karla Nogueira Pestana ${ }^{3}$ \\ Juliana da Silva Alves ${ }^{4}$, Dreid de Cerqueira Silveira ${ }^{4}$
}

\begin{abstract}
RESUMO
$\mathrm{Na}$ bananeira ocorrem variações somaclonais em taxa muito superior ao que se observa na maioria das outras culturas, provavelmente em função da instabilidade mitótica. Objetivou-se com o presente trabalho avaliar clones de bananeira Cavendish coletados em diferentes locais. Os clones Grande Naine (G.N. Taperão, G.N. Rossete, G.N. Williams, G.N. Magário, G.N. SC074) e Nanicão (N. IAC Abóbora Verde, N. Rossete, N. SC-0008 e N. SC-063) coletados em São Paulo, Santa Catarina e Bahia foram avaliados no Lote 54-P da Thelo Produção Agropecuária (Grupo Plena), no Projeto Jaíba, Etapa 1, no município de Matias Cardoso-MG. Avaliaram-se os caracteres altura da planta, circunferência do pseudocaule, número de folhas vivas no florescimento e na colheita, número de brotos, peso do cacho e das pencas, número de frutos e de pencas por cacho, comprimento e circunferência do fruto e número de dias do plantio ao florescimento e à colheita. Observou-se grande similaridade nas características dos clones. No entanto, os resultados obtidos permitem a recomendação dos clones N. IAC Abóbora Verde e G.N. Williams.
\end{abstract}

Termos para indexação: Musa spp., variabilidade, mutações espontâneas.

\begin{abstract}
Somaclonal variations occur in bananas at greater rates compared to other crops, probably due to mitotic instability. The objective of the present research was to evaluate Cavendish banana clones collected from different sites. The 'Grand Naine' clones (G.N. Taperão, G.N. Rossete, G.N. Williams, G.N. Magário, G.N. SC-074) and 'Nanicão' (N. IAC Abóbora Verde, N. Rossete, N. SC-0008 and N. SC-063) collected from the states of São Paulo, Santa Catarina and Bahia, were evaluated at the Station 54-P of the Thelo Agricultural Production (Plena Group), in the Jaíba Project, Stage 1, in the city of Matias Cardoso-MG. The characteristics of the plant as height and pseudostem circumference, number of live leaves at flowering and harvesting, number of shoots, weight of bunch and hand, number of fruits and hands per bunch, fruit length and circumference, and the number of days from planting to flowering and harvest, were evaluated. Great similarity among the clone characteristics was observed. However, the results allowed to recommend the following clones: N. IAC Abóbora Verde and G.N. Williams.
\end{abstract}

Index terms: Musa spp., variability, spontaneous mutations.

(Recebido para publicação em 4 de outubro de 2005 e aprovado em 12 de maio de 2006)

\section{INTRODUÇÃO}

A bananeira é uma planta tipicamente tropical, exigindo calor constante, elevada umidade relativa (>80\%) e precipitações bem distribuídas (100 a $180 \mathrm{~mm} / \mathrm{mês}$ ) para o seu bom desenvolvimento e produção. Embora esses fatores climáticos limitem a área de produção, o Brasil apresenta condições favoráveis ao cultivo da bananeira em quase todo seu território.

A maioria das cultivares de banana originou-se no continente asiático, embora existam centros secundários de origem na África Oriental e nas ilhas do Pacífico. As cultivares apresentam três níveis cromossômicos distintos: diplóide, triplóide e tetraplóide, respectivamente com dois, três e quatro conjuntos de 11 cromossomos. Na evolução das bananeiras comestíveis participaram, principalmente, as espécies diplóides selvagens Musa acuminata Colla (AA) e Musa balbisiana Colla (BB).

Conhecer a situação dos recursos fitogenéticos de um país ou região é da maior importância para se avaliar acessos, resolver sinonímia, identificar mutantes e classificar cultivares de acordo com o genoma, utilizando critérios morfotaxonômicos. Atenção especial deve ser prestada à prospecção, coleta e conservação dos clones para evitar a erosão da reserva genética e assegurar sua utilização nos programas de melhoramento (SINGH \& CHADLA, 1996).

\footnotetext{
${ }^{1}$ Engenheiro Agrônomo, D.Sc., Pesquisador da Embrapa Mandioca e Fruticultura - Cx. P. 007 - 44380-000 - Cruz das Almas, BA ssilva@cnpmf.embrapa.br

${ }^{2}$ Engenheiro Agrônomo, M.Sc., Sócio-Diretor da Plena Consultoria e Projetos - Rua Teixeira de Freitas 478, Salas 907 - Santo Antônio - 30350-180 Belo Horizonte, MG.

${ }^{3}$ Bolsista de IC PIBIC - Embrapa Mandioca e Fruticultura Tropical - Cx. P. 007 - 44.380-000 - Cruz das Almas, BA - karlapestana6@yahoo.com.br ${ }^{4}$ Bolsista de IC FAPESB - Embrapa Mandioca e Fruticultura Tropical - Cx. P. 007 - 44.380-000 - Cruz das Almas, BA - jualvesagr@yahoo.com.br; suely@cnpmf.embrapa.br
} 
$\mathrm{Na}$ bananeira ocorrem variações somaclonais em taxa muito superior ao que se observa na maioria das outras culturas, provavelmente em função da instabilidade mitótica (mitose que resulta na produção de células com diferentes ploidias), que não é exclusiva da cultura de tecidos, sendo também observada em campo, porém em frequiências menores (HWANG \& TANG, 1996). Desta forma, pode ser explicado o aparecimento de dezenas de cultivares do grupo genômico (AAA), subgrupo Cavendish, e de cultivares AAB como a 'Pacovan', mutante da 'Prata'. Mediante a avaliação do germoplasma pode-se obter produtividade e resistência às pragas e doenças, a exemplo das cultivares Caipira e Thap Maeo recomendadas aos agricultores (SILVA et al., 2002).

O futuro do melhoramento e/ou manutenção da cadeia produtiva da banana implica não só a exploração de diferentes tipos/cultivares na geração de híbridos, mas também o uso da variabilidade genética dentro dos diversos tipos (SHEPHERD, 1994). Assim, cada cultivar de banana tem suas próprias vantagens e desvantagens e pode gerar mais ou menos variantes.

Os produtores devem levar em conta que a solução para seus problemas de produção podem não residir apenas nos genótipos melhorados. Os enfoques holísticos de manejo integrado dos cultivos também têm muito o que oferecer (DANIELLS, 2000).

Foram coletados em São Paulo, Minas Gerais, Bahia e Santa Catarina, 69 clones Cavendish, sendo 49 do tipo 'Nanicão' e 20 do tipo 'Grande Naine' (FERREIRA \& SILVA, 2002). Uma primeira avaliação foi feita em Cruz das Almas, na Embrapa Mandioca e Fruticultura Tropical, considerando: altura de plantas, ciclo, peso de cacho, número e comprimento de frutos. Os resultados obtidos demonstraram variabilidade em todos os caracteres avaliados (SILVA et al., 2004). Selecionaram-se alguns clones de Grande Naine e Nanicão (subgrupo Cavendish), que foram avaliados sob as condições da grande região produtora de banana no Norte de Minas Gerais, constituindo-se o objetivo deste trabalho.

\section{MATERIAL E MÉTODOS}

Foram avaliados em Cruz das Almas-BA, 69 clones de Cavendish coletados nos Estados de São Paulo, Minas Gerais, Bahia e Santa Catarina, sendo 49 do tipo Nanicão e 20 do tipo Grande Naine (FERREIRA \& SILVA, 2002). Dentre estes materiais avaliados, foram selecionados clones de Grande Naine (G.N. Taperão, G.N. Rossete, G.N. Williams, G.N. Magário, G.N. SC-074) e Nanicão (N. IAC Abóbora Verde, N. Rossete, N. SC-0008 e N. SC-063) (Tabela 1). Estes clones, juntamente com uma testemunha (Grande Naine-GN) foram reavaliados no Norte de Minas Gerais, em experimento instalado no Lote 54-P da Thelo Produção Agropecuária (Grupo Plena), no Projeto Jaíba, Etapa 1, no município de Matias Cardoso-MG.

A região é caracterizada por clima tropical de savana (classificação de Köppen), com pluviosidade média anual de $930 \mathrm{~mm}$ e temperatura média na faixa de 22 a $26^{\circ} \mathrm{C}$, com insolação média anual de 232,8 horas de sol por mês (BRASIL, 1992).

TABELA 1 - Acessos de germoplasma de banana, coletados nos Estados de São Paulo, Bahia e Santa Catarina.

\begin{tabular}{llllc}
\hline \multicolumn{1}{c}{ Nome $^{\mathbf{1}}$} & \multicolumn{1}{c}{ Município } & \multicolumn{1}{c}{ Lat. } & Long. & Alt. \\
\hline G.N. Rossete & Jacupiranga-SP & $24^{\circ} 45^{\prime} \mathrm{S}$ & $48^{\circ} 00^{\prime} \mathrm{W}$ & 25 \\
G.N. Magário & Sete Barras-SP & $24^{\circ} 21^{\prime} \mathrm{S}$ & $47^{\circ} 56^{\prime} \mathrm{W}$ & 25 \\
G.N. SC-074 & Miracatú-SP & $24^{\circ} 16^{\prime} \mathrm{S}$ & $47^{\circ} 28^{\prime} \mathrm{W}$ & 27 \\
G.N. Taperão & Brotas-SP & $22^{\circ} 14^{\prime} \mathrm{S}$ & $48^{\circ} 13$ ' $\mathrm{W}$ & 520 \\
G.N. Williams & Cruz das Almas-BA & $12^{\circ} 44^{\prime} \mathrm{S}$ & $39^{\circ} 06^{\prime} \mathrm{W}$ & 220 \\
Grande Naine & Cruz das Almas-BA & $12^{\circ} 44^{\prime} \mathrm{S}$ & $39^{\circ} 06^{\prime} \mathrm{W}$ & 220 \\
N. Rossete & Jacupiranga-SP & $24^{\circ} 45^{\prime} \mathrm{S}$ & $48^{\circ} 00^{\prime} \mathrm{W}$ & 25 \\
N. SC-008 & Jacinto Machado-SC & $29^{\circ} 00^{\prime} \mathrm{S}$ & $49^{\circ} 46^{\prime} \mathrm{W}$ & 50 \\
NSC-063 & Miracatú-SP & $24^{\circ} 16^{\prime} \mathrm{S}$ & $47^{\circ} 28^{\prime} \mathrm{W}$ & 27 \\
N. IAC Abóbora Verde & Pariquera-Açu-SP & $24^{\circ} 44^{\prime} \mathrm{S}$ & $47^{\circ} 56^{\prime} \mathrm{W}$ & 20 \\
\hline
\end{tabular}

${ }^{1} \mathrm{~N}$ :Nanicão; GN: Grande Naine. 
O solo predominante é o Latossolo Amarelo Distrófico, textura arenosa/média leve, álico, com baixa capacidade de retenção de umidade e fertilidade natural (SOUZA et al., 1999). No local, em 1999, foi cultivada a bananeira 'Prata Anã', que foi erradicada por causa do ataque do mal-do-Panamá (Fusarium oxysporum).

O experimento foi conduzido de janeiro de 2004 a janeiro de 2005. O delineamento foi em blocos casualizados com cinco repetições. A parcela constou de cinco plantas, dispostas no espaçamento em fileiras duplas de $3,60 \mathrm{~m} \mathrm{x}$ $2,40 \mathrm{~m}$ x 2,00 m. A densidade de plantio foi de 1.667 plantas/ ha. Como bordadura lateral, utilizou-se a cultivar Thap Maeo.

Os requerimentos agronômicos relativos a preparo do solo, calagem, abertura de covas, adubação, plantio, práticas culturais e tratamentos fitossanitários foram realizados conforme preconizados para o cultivo da bananeira (BORGES \& SOUZA, 2004).

$\mathrm{Na}$ avaliação, foram consideradas as seguintes variáveis: altura da planta em centímetro (ALP), circunferência do pseudocaule em centímetro (CPC), número de folhas vivas no florescimento (FVF), número de folhas vivas na colheita (FVC), número de brotos (NBT), número de dias do plantio ao florescimento (DPF), número de dias do plantio à colheita (DPC), peso do cacho em quilogramas (PCA), peso das pencas em quilograma (PPE), número de frutos por cacho (NFC), número de pencas por cacho (NPE), comprimento do fruto em centímetro (COF) e circunferência do fruto em centímetro (CIF).

\section{RESULTADOS E DISCUSSÃO}

Na Tabela 2 são apresentadas as médias de altura de planta (ALP), circunferência de pseudocaule (CPC), número de folhas vivas na inflorescência (FVF) e colheita $(\mathrm{FVC})$, número de brotos (NBT) e de dias do plantio à inflorescência. Como pode-se observar há uma grande uniformidade nas características dos clones. Para altura de planta, circunferência do pseudocaule e número de folhas vivas na colheita houve formação de dois grupamentos, nos demais caracteres não houve formação de grupos pelo Teste Scott-Knott.

A altura de planta variou de $280,00 \mathrm{~cm}$ no clone $\mathrm{N}$. IAC Abóbora Verde a 253,00 cm no clone G. N. Rossete. Como esperado, a maioria dos clones 'Grande Naine', apresentou porte menor que os clones 'Nanicão'. Houve a formação de dois grupamentos pelo teste Scott-Knott a $5 \%$. Entre o primeiro e segundo grupamentos houve uma diferença percentual de 6,58\%. Não se obteve clones de porte muito baixo, embora a busca de plantas baixas e produtivas tenha sido um dos objetivos da coleta. A maioria dos clones avaliados apresentou altura superior a 200,00 cm (FERREIRA \& SILVA, 2002). Estes mesmos clones avaliados em Cruz das Almas apresentaram, no primeiro ciclo, uma altura que variou de $231,00 \mathrm{~cm}$ para o genótipo N. IAC Verde a $189,70 \mathrm{~cm}$ no genótipo G. N. Taperão. Em média, os valores obtidos neste trabalho foram superiores aos observados em Cruz das Almas (SILVA et al., 2004).

TABELA 2 - Médias dos caracteres observados na época do florescimento de dez clones de banana Cavendish, no primeiro ciclo de produção. Matias Cardoso, MG, Janeiro 2005¹.

\begin{tabular}{lccccccc}
\hline \multirow{2}{*}{ Genótipos } & \multicolumn{7}{c}{ Características $^{2}$} \\
\cline { 2 - 8 } & ALP & CPC & FVF & FVC & NBT & DPF & DPC \\
\hline N. IAC A. Verde & $280,00 \mathrm{a}$ & $81,83 \mathrm{~b}$ & $15,13 \mathrm{a}$ & $10,31 \mathrm{a}$ & $8,96 \mathrm{a}$ & $249,31 \mathrm{a}$ & $359,67 \mathrm{a}$ \\
N. SC-063 & $279,00 \mathrm{a}$ & $85,80 \mathrm{a}$ & $14,86 \mathrm{a}$ & $9,90 \mathrm{a}$ & $9,43 \mathrm{a}$ & $272,43 \mathrm{a}$ & $364,27 \mathrm{a}$ \\
N. Rossete & $277,00 \mathrm{a}$ & $85,16 \mathrm{a}$ & $15,36 \mathrm{a}$ & $10,00 \mathrm{a}$ & $9,60 \mathrm{a}$ & $250,20 \mathrm{a}$ & $357,37 \mathrm{a}$ \\
N. SC-0008 & $275,00 \mathrm{a}$ & $83,50 \mathrm{a}$ & $14,63 \mathrm{a}$ & $9,83 \mathrm{a}$ & $8,50 \mathrm{a}$ & $245,47 \mathrm{a}$ & $360,67 \mathrm{a}$ \\
G. N. SC-074 & $271,00 \mathrm{a}$ & $84,40 \mathrm{a}$ & $15,00 \mathrm{a}$ & $9,70 \mathrm{a}$ & $8,80 \mathrm{a}$ & $245,33 \mathrm{a}$ & $361,09 \mathrm{a}$ \\
Grande Naine & $255,00 \mathrm{~b}$ & $81,26 \mathrm{~b}$ & $14,50 \mathrm{a}$ & $8,86 \mathrm{~b}$ & $8,27 \mathrm{a}$ & $249,87 \mathrm{a}$ & $359,67 \mathrm{a}$ \\
G. N. Williams & $264,00 \mathrm{~b}$ & $85,20 \mathrm{a}$ & $14,76 \mathrm{a}$ & $9,80 \mathrm{a}$ & $8,60 \mathrm{a}$ & $238,37 \mathrm{a}$ & $357,37 \mathrm{a}$ \\
G. N. Magario & $260,00 \mathrm{~b}$ & $80,00 \mathrm{~b}$ & $14,30 \mathrm{a}$ & $8,93 \mathrm{~b}$ & $8,33 \mathrm{a}$ & $243,30 \mathrm{a}$ & $363,30 \mathrm{a}$ \\
G. N. Taperão & $259,00 \mathrm{~b}$ & $81,93 \mathrm{~b}$ & $14,30 \mathrm{a}$ & $9,56 \mathrm{a}$ & $8,97 \mathrm{a}$ & $242,97 \mathrm{a}$ & $359,63 \mathrm{a}$ \\
G. N. Rossete & $253,00 \mathrm{~b}$ & $81,50 \mathrm{~b}$ & $14,60 \mathrm{a}$ & $9,33 \mathrm{~b}$ & $9,00 \mathrm{a}$ & $244,00 \mathrm{a}$ & $361,67 \mathrm{a}$ \\
CV\% & 3,57 & 3,35 & 4,49 & 5,49 & 9,87 & 7,00 & 2,37 \\
\hline
\end{tabular}

${ }^{1}$ Médias seguidas pela mesma letra nas colunas pertencem ao mesmo grupo pelo teste de agrupamento de Scott-Knott a $5 \%$ de probabilidade.

${ }^{2}$ Altura da planta (ALP) - cm; circunferência do pseudocaule (CPC) - cm; folhas vivas no florescimento (FVF); folhas vivas na colheita (FVC); número de brotos (NBT); número de dias do plantio ao florescimento (DPF); número de dias do plantio à colheita (DPC).

Ciênc. agrotec., Lavras, v. 30, n. 5, p. 832-837, set./out., 2006 
A altura da planta é um descritor importante do ponto de vista fitotécnico e de melhoramento, pois influi nos aspectos de densidade de plantio e manejo da cultura, interferindo na produção (BELALCÁZARCARVAJAL, 1991).

A circunferência do pseudocaule apresentou variação de 85,80 cm (N. SC-063) a 80,00 cm (G.N. Magário). Foram identificados dois agrupamentos para este caráter no ciclo de produção avaliado (Tabela 2). Os genótipos do grupo dois apresentaram circunferência do pseudocaule $4,13 \%$ inferior aos do grupo um. A maioria dos clones da cultivar Grande Naine apresentou menor circunferência quando comparadas aos da cultivar Nanicão, exceto o $\mathrm{N}$. IAC Abóbora Verde, classificado no segundo agrupamento e os clones G. N. Williams e G. N. SC-074 classificados no primeiro agrupamento.

O tombamento de plantas e/ou a quebra do pseudocaule pela ação dos ventos estão relacionados com porte e perímetro do pseudocaule da planta. No entanto, o tombamento ocorre com maior freqüência quando ocorre baixo vigor do sistema radicular, retirada de mudas, ataque de broca-do-rizoma, nematóides e afloramento do rizoma, enquanto a quebra é devida, principalmente, aos aspectos nutricionais, de irrigação e resistência do tecido foliar que forma o pseudocaule (TEIXEIRA, 2001).

Os clones não apresentaram diferença com relação ao número de folhas vivas na inflorescência. No entanto, para o número de folhas vivas na colheita houve a formação de dois agrupamentos, onde os genótipos do segundo grupo (Grande Naine, Grande Naine Magário e Grande Naine Rossete) apresentaram uma redução de $8,41 \%$ em relação aos do primeiro grupo (N. IAC. Abóbora Verde, N. SC-063, N. Rossete, N. SC-0008, G.N. SC-074, G.N. Williams e G. N. Taperão). A variação foi de 10,31 (N. IAC A. Verde) a 9,33 (G. N. Rossete (Tabela 2). Como pode ser observado, todos os clones que apresentaram menor número de folhas vivas na colheita eram da 'Grande Naine'. O número de folhas vivas é importante na avaliação de cultivares, pois poderá influenciar o desenvolvimento do cacho, o qual dependerá diretamente da taxa de fotossíntese da planta (ALVES, 1999). A redução do número de folhas da floração até a colheita deve-se, sobretudo, à translocação de fotoassimilados para a formação de frutos, que passam a ser o dreno preferencial da planta, à senescência natural das folhas e à presença de Sigatokaamarela. Sabe-se que para o enchimento de frutos em Cavendish são necessárias no mínimo oito folhas ativas na colheita, portanto, todos os genótipos apresentaram valores ideais de folhas (SOTO-BALLESTERO, 1992).
Com relação ao número de brotos, os genótipos encontram-se no mesmo grupo, não ocorrendo nenhuma diferença estatística entre eles. Uma produção satisfatória de brotos pode ser desejável caso se queira multiplicar o genótipo em campo. Teoricamente, uma planta tem a capacidade de produzir tantos rebentos quanto forem as folhas emitidas. No entanto, o aparecimento de novos filhos parece ser influenciado pela dominância apical da planta-mãe e de outros filhos em desenvolvimento. A cultivar, altura e idade da planta-mãe são fatores importantes na determinação do número de rebentos emitidos (SOTO-BALLESTERO, 1992).

De forma semelhante ao ocorrido em DPF, não houve diferença estatística entre os genótipos para DPC. Embora não tenha havido grande diferença nos ciclos dos clones entre este trabalho e o realizado por Silva et al. (2004), vale ressaltar que, em Cruz das Almas, os mesmos genótipos foram classificados em dois agrupamentos, quanto ao número de dias do plantio à colheita, com variações de 358,70 para o genótipo N. IAC Verde a 340,70 para o G. N. SC-074. O ciclo é um caráter de relevância na cultura da bananeira, já que reflete a precocidade da planta, e a redução do número de dias necessários à emissão do cacho é desejada, pois representa a antecipação do investimento aplicado na lavoura.

Os resultados apresentados na Tabela 3 revelaram grande uniformidade entre os genótipos com relação aos caracteres número de frutos, comprimento do fruto, circunferência do fruto e número de pencas.

Com relação ao peso do cacho e ao peso das pencas houve a formação de dois grupamentos de genótipos. O primeiro formado pelos clones N. SC-063, N. Rossete, G. N. Williams, G. N. Rossete, N. IAC A. Verde, G. N. Taperão, G. N. SC-074 e G. N. Magário. O segundo grupo foi constituído pelo N. SC-0008 e Grande Naine testemunha. O PCA teve uma variação de $37,73 \mathrm{~kg}$ (NSC-063) a 32,39 $\mathrm{kg}$ (G.N. Testemunha) e o PPE variou de 34,62 kg para o clone N.SC-063 a 29,34 kg para a Grande Naine Testemunha. O PCA do primeiro grupo foi $8,77 \%$ superior ao do segundo grupo. Os descritores peso do cacho e peso das pencas apresentam estreita relação entre si, uma vez que a diferença entre eles corresponde ao peso do engaço, uma estrutura que não varia muito entre as cultivares do mesmo subgrupo. Em trabalhos realizados anteriormente com os mesmos genótipos, observou-se que o peso de cacho do segundo ciclo foi pouco superior $(1,85 \%)$ aos valores obtidos no primeiro ciclo, embora todos os genótipos tenham sido classificados no mesmo grupamento no primeiro e no segundo ciclos, à exceção da Grande Naine, testemunha que ficou em um grupamento inferior (SILVA et al., 2004). 
Apesar do peso de cacho estar diretamente relacionado ao número de frutos, comprimento e circunferência dos frutos e número de pencas, para peso de cacho houve formação de dois grupamentos e em todos os outros caracteres não se formaram grupos de clones.

$\mathrm{Na}$ avaliação realizada em Cruz das Almas, por Silva et al. (2004), os números de frutos (NFC) observados nos mesmos genótipos apresentaram variação de 163,00 no G. N. Taperão e de 131,00 para a G. N. Williams, com os genótipos classificados em diferentes grupos. Quanto ao comprimento dos frutos não houve formação de agrupamentos de genótipos. Os valores de peso de cacho, comprimento e número de frutos observados neste trabalho foram bastante superiores aos obtidos por Silva et al. (2004). Isto, provavelmente, foi devido às condições de cultivo do Norte de Minas serem mais favoráveis para produção de banana que as de Cruz das Almas e incluir no seu sistema de produção a eliminação do coração, operação não realizadas em Cruz das Almas.

O comprimento e a circunferência do fruto, mais adequados à exportação, são, respectivamente, de $22 \mathrm{~cm}$ e 10,01 cm (FRUTISSÉRIES, 2000). Como pode-se observar, a maioria dos clones atingiu o comprimento de frutos e todos os genótipos apresentaram circunferência de frutos superiores ao exigido para a exportação. Um dos fatores que poderiam ter favorecido a ocorrência de frutos grandes foi a eliminação do coração e o desbaste das últimas pencas, uma vez que o emprego destas práticas podem levar a um incremento do tamanho do fruto e conseqüentemente um aumento do peso do cacho (SOTOBALLESTERO, 1992).

O caráter número de pencas por cacho é de importância para produtor, uma vez que grande parte do mercado usa a penca como unidade comercial, além do que, um aumento no número de pencas acarreta um aumento no número de frutos e, consequentemente, uma elevação do peso do cacho (SOTO-BALLESTERO, 1992).

A tarefa de selecionar os melhores clones em um grupo tão uniforme como o estudado não é fácil. Assim, o critério usado na eliminação de um clone foi qualquer caráter indesejável, por ele apresentado. Desta forma os clones N. SC-008 e Grande Naine Testemunha foram descartados pelo baixo PCA, inferior aos demais, o G.N. SC-074 pela altura elevada da planta e o G.N. Rossete pelo reduzido número de folhas vivas na colheita.

$O$ reduzido desenvolvimento dos seguidores (plantas do segundo ciclo) levou à eliminação dos clones N. SC-063, N. Rossete e G.N. Taperão, o que no mínimo resultaria em um aumento do número de dias para colheita do segundo ciclo.

TABELA 3 - Médias dos caracteres observados na época da colheita de dez clones de banana Cavendish, no primeiro ciclo de produção. Matias Cardoso, MG, Janeiro $2005^{1}$.

\begin{tabular}{lllllll}
\hline \multirow{2}{*}{ Genótipos } & \multicolumn{7}{c}{ Características $^{2}$} \\
\cline { 2 - 7 } & PCA & PPE & NFC & COF & CIF & NPE \\
\hline N. SC-063 & $37,73 \mathrm{a}$ & $34,62 \mathrm{a}$ & $170,37 \mathrm{a}$ & $22,81 \mathrm{a}$ & $13,60 \mathrm{a}$ & $9,70 \mathrm{a}$ \\
N. Rossete & $37,42 \mathrm{a}$ & $34,14 \mathrm{a}$ & $168,33 \mathrm{a}$ & $23,09 \mathrm{a}$ & $13,49 \mathrm{a}$ & $9,50 \mathrm{a}$ \\
G. N. Williams & $36,60 \mathrm{a}$ & $33,45 \mathrm{a}$ & $166,80 \mathrm{a}$ & $22,13 \mathrm{a}$ & $13,90 \mathrm{a}$ & $9,53 \mathrm{a}$ \\
G. N. Rossete & $36,16 \mathrm{a}$ & $32,94 \mathrm{a}$ & $171,77 \mathrm{a}$ & $22,81 \mathrm{a}$ & $13,43 \mathrm{a}$ & $9,77 \mathrm{a}$ \\
N. IAC A. Verde & $36,03 \mathrm{a}$ & $32,94 \mathrm{a}$ & $167,35 \mathrm{a}$ & $22,36 \mathrm{a}$ & $13,35 \mathrm{a}$ & $9,37 \mathrm{a}$ \\
G. N. Taperão & $36,00 \mathrm{a}$ & $32,66 \mathrm{a}$ & $168,47 \mathrm{a}$ & $22,99 \mathrm{a}$ & $13,38 \mathrm{a}$ & $9,80 \mathrm{a}$ \\
G. N. SC-074 & $36,00 \mathrm{a}$ & $32,91 \mathrm{a}$ & $168,23 \mathrm{a}$ & $22,44 \mathrm{a}$ & $13,50 \mathrm{a}$ & $9,70 \mathrm{a}$ \\
G. N. Magário & $35,94 \mathrm{a}$ & $32,68 \mathrm{a}$ & $172,10 \mathrm{a}$ & $22,72 \mathrm{a}$ & $13,52 \mathrm{a}$ & $9,63 \mathrm{a}$ \\
N. SC-0008 & $34,17 \mathrm{~b}$ & $30,93 \mathrm{~b}$ & $162,17 \mathrm{a}$ & $22,40 \mathrm{a}$ & $13,13 \mathrm{a}$ & $9,43 \mathrm{a}$ \\
Grande Naine & $32,39 \mathrm{~b}$ & $29,34 \mathrm{~b}$ & $161,87 \mathrm{a}$ & $21,33 \mathrm{a}$ & $13,11 \mathrm{a}$ & $9,35 \mathrm{a}$ \\
\hline CV\% & 6,14 & 6,43 & 4,44 & 4,26 & 2,97 & 4,41
\end{tabular}

${ }^{1}$ Médias seguidas pela mesma letra nas colunas pertencem ao mesmo grupo pelo teste de agrupamento de Scott-Knott a $5 \%$ de probabilidade.

${ }^{2}$ Peso do cacho (PCA) em (kg); peso das pencas (PPE) em (kg); número de fruto (NFC); comprimento do fruto (COF) em $(\mathrm{cm})$; circunferência do fruto $(\mathrm{CIF})$ em $(\mathrm{cm})$; número de pencas (NPE).

Ciênc. agrotec., Lavras, v. 30, n. 5, p. 832-837, set./out., 2006 
Portanto, considerou-se como os melhores clones o N. IAC Abóbora Verde e o G.N. Williams. Embora o primeiro ciclo não seja o mais adequado para fazer seleção, a baixa diferença de produção observada entre o primeiro e segundo ciclos $(1,85 \%)$ nestes clones avaliados em Cruz das Almas (SILVA et al., 2004) e o baixo desenvolvimento dos seguidores observado em alguns clones, favoreceu esta tomada de decisão.

\section{CONCLUSÃO}

Considerando o desempenho dos clones com relação aos caracteres avaliados recomendam-se os clones N. IAC Abóbora Verde e G.N. Williams.

\section{AGRADECIMENTOS}

Ao Técnico Agrícola Airto Gonçalves Dutra pelo apoio na condução dos trabalhos de campo, na Thelo Produção Agropecuária (Grupo PLENA), no Projeto Jaíba, Etapa 1, no município de Matias Cardoso-MG.

\section{REFERÊNCIAS BIBLIOGRÁFICAS}

ALVES, E. J. (Org.). A cultura da banana: aspectos técnicos socioeconômicos e agroindustriais. 2. ed. Brasília, DF: SPI; Cruz das Almas: Embrapa-CNPMF, 1999. 585 p.

BELALCÁZAR-CARVAJAL, S. L. El cultivo del plátano en el trópico. Cali: Feriva, 1991. 376 p.

BORGES, A. L.; SOUZA, L. da S. $O$ cultivo da bananeira. Cruz das Almas: Embrapa Mandioca e Fruticultura, 2004. $279 \mathrm{p}$.

BRASIL. Ministério da Agricultura, Pecuária e Abastecimento. Departamento Nacional de Meteorologia. Normas Climatológica: 1961-1990. Brasília, DF, 1992. 84 p.

DANIELLS, J. Qué variedad de banano debo cultivar? Informusa, Montpellier, v. 9, n. 1, p. 31-33, jun. 2000.

FERREIRA, F. R.; SILVA, S. O. e. E. Collecting banana germplasm from the AAA genomic group/Cavendish subgroup. Crop Breeding and Applied Biotechnology, Londrina, v. 2, n. 3, p. 485-488, 2002.

FRUTISSÉRIES. Banana. Brasília, DF: MI, 2000.

HWANG, S. C.; TANG, C. Y. Somaclonal variation and its use for Improving Cavendish (AAA desert) bananas in Taiwan. In: NEW FRONTIERS IN RESISTANCE FOR NEMATODE FUSARIUM AND SIGATOKA, 1., 1995, Kuala Lummpur, Malaysia. Proceedings... Montpellier: Inibap, 1996. p. 173-181.

SHEPHERD, K. Mejoramiento de bananos: historia y metodos. Informusa, Montpellier, v. 3, n. 1, p. 10-11, jun. 1994.

SILVA, S. O.; ALVES, E. J.; ANDRADE NETO, T. M.; LICHTEMBERG, L. A.; FERREIRA, F. R. Avaliação de clones de bananeira do subgrupo Cavendish (Musa acuminata, AAA) em Cruz das Almas-BA. Ciência e Agrotecnologia, Lavras, v. 28, n. 6, p. 1247-1258, 2004.

SILVA, S. O. e; ALVES, E. J.; LIMA, M. B.; SILVEIRA, J. R. S. Bananeira. In: BRUCKNER, C. H. (Org.). Melhoramento de fruteiras tropicais. Viçosa: UFV, 2002. v. 1, p. 101-157.

SINGH, H. P.; CHADLA, K. L. Bananos y plátanos en la Índia. Informusa, Montpellier, v. 5, n. 2, p. 22-25, dic. 1996.

SOTO-BALLESTERO, M. Bananos: cultivo y comercialización. 2. ed. San José: Lil, 1992. 674 p.

SOUZA, L. da S.; BORGES, A. L.; SILVA, J. T. A. da. Características físicas e químicas de solos cultivados com bananeira, sob irrigação, na região norte de Minas Gerais. Cruz das Almas: Embrapa Mandioca e Fruticultura, 1999. 24 p. (Boletim de pesquisa, 14).

TEIXEIRA, L. A. J. Cultivares de bananeira. In: RUGGIERO, C. (Coord.). Bananicultura. Jaboticabal: FUNEP, 2001. p. 150-170. 